Sur un test d'égalité des autocovariances de deux séries chronologiques

Author(s): Guy Melard and Roch Roy

Source: The Canadian Journal of Statistics / La Revue Canadienne de Statistique, Vol. 12, No. 4 (Dec., 1984), pp. 333-342

Published by: Statistical Society of Canada

Stable URL: http://www.jstor.org/stable/3314816

Accessed: 10-12-2017 11:26 UTC

JSTOR is a not-for-profit service that helps scholars, researchers, and students discover, use, and build upon a wide range of content in a trusted digital archive. We use information technology and tools to increase productivity and facilitate new forms of scholarship. For more information about JSTOR, please contact support@jstor.org.

Your use of the JSTOR archive indicates your acceptance of the Terms \& Conditions of Use, available at http://about.jstor.org/terms 


\title{
Sur un test d'égalité des autocovariances de deux séries chronologiques*
}

\author{
Guy MELARD et Roch ROY
}

Université Libre de Bruxelles et Université de Montréal

Key words and phrases: Comparison of autocovariance functions, stationary time series, estimation of the covariance matrix of the sample autocovariances.

AMS 1980 subject classifications: Primary 62M10.

\section{RÉSUMÉ}

Nous proposons un test d'égalité des fonctions d'autocovariances de deux séries chronologiques stationnaires et indépendantes. Le test est basé sur une forme quadratique du vecteur des différences des $\mathbf{J}+1$ premières autocovariances. La distribution asymptotique de la statistique considérée est obtenue sous l'hypothèse nulle et les propriétés du test, notamment le biais et la puissance, sont examinés pour des séries finies par des simulations de Monte Carlo. La statistique utilisée fait intervenir un nouvel estimateur de la structure de covariance des autocovariances échantillonnales qui fournit une matrice de covariance définie positive. Nous montrons que cet estimateur est convergent au sens de la norme $L_{1}$.

\section{ABSTRACT}

We propose a test for the equality of the autocovariance functions of two independent and stationary time series. The test statistic is a quadratic form in the vector of differences of the first $J+1$ autocovariances. Its asymptotic distribution is derived under the null hypothesis, and the finite-sample properties of the test, namely the bias and the power, are investigated by Monte Carlo methods. A by-product of this study is a new estimator of the covariance between two sample autocovariances which provides a positive definite covariance matrix. We establish the convergence of this estimator in the $L_{1}$ norm.

\section{INTRODUCTION}

Lors de l'analyse de deux ou plusieurs séries chronologiques, il est naturel de se demander si ces dernières ont été engendrées par le même processus. Dans cet article, nous proposons une méthode non-paramétrique permettant de tester l'égalité des fonctions d'autocovariances de deux séries stationnaires et indépendantes. La distribution asymptotique des autocovariances échantillonnales est connue depuis fort longtemps et la structure de covariance est donnée par la formule de Bartlett (Anderson 1971, p. 481). Cependant, nous devons estimer la matrice de covariance des autocovariances échantillonnales. Ce problème a déjà été étudié notamment par Robinson (1977) selon l'approche temporelle et par Brillinger (1978, 1981) ainsi que par Taniguchi (1982) selon l'approche fréquentielle. Comme notre travail se situe dans l'approche temporelle, nous avons envisagé l'utilisation de l'estimateur de Robinson. Cependant cet estimateur ne

\footnotetext{
*Ce projet a été subventionné partiellement par le Conseil de recherche en sciences naturelles et en génie du Canada ainsi que par la Coopération internationale belgo-québécoise.
} 
fournit pas nécessairement une matrice de covariance définie positive. Dans notre cas, la positivité de la matrice de covariance estimée est particulièrement importante puisque la statistique proposée fait intervenir une forme quadratique basée sur l'inverse de cette matrice. Ceci nous a amené à définir un nouvel estimateur de la covariance entre deux autocovariances échantillonnales qui fournit une matrice de covariance définie positive. Nous montrons que cet estimateur est convergent au sens de la norme $L_{1}$.

Le test introduit dans cet article peut servir non seulement à comparer la structure d'autocovariance de deux séries chronologiques mais aussi à vérifier la stabilité du processus générateur d'une série unique. Dans la mesure où la période d'observations est suffisamment longue, il semble raisonnable de la partager en deux et, en laissant de côté quelques valeurs intermédiaires, les deux sous-séries peuvent être considérées comme indépendantes et le test s'applique. Le test proposé dans cet article ne nécessite l'estimation d'aucun modèle, il est donc non-paramétrique et s'insère dans la batterie des techniques statistiques utilisables à l'étape d'identification dans le processus itératif de construction d'un modèle de série chronologique selon la méthode de Box et Jenkins (1976).

Le contenu de l'article est le suivant. Dans la Section 2, nous introduisons la statistique définissant le test et déterminons sa distribution asymptotique. A la Section 3, nous proposons un nouvel estimateur de la covariance de deux autocovariances échantillonnales et nous montrons qu'il converge au sens de la norme $L_{1}$. Dans la dernière section, nous étudions le comportement du test pour des séries finies par la méthode de Monte Carlo. Pour quelques modèles simples, le biais et la puissance sont évalués.

\section{DISTRIBUTION DE LA STATISTIQUE PROPOSÉE}

Soit $X_{k t}, t=1, \ldots, n_{k}$ une réalisation finie d'un processus stationnaire $X_{k}$ avec moments d'ordre 1 et 2

$$
\mu_{k}=\mathscr{E}\left[X_{k t}\right], \quad \gamma_{k j}=\mathscr{E}\left[\left(X_{k t}-\mu_{k}\right)\left(X_{k, t+j}-\mu_{k}\right)\right]
$$

où $k=1,2$ et dont tous les cumulants d'ordre 4 sont nuls et tel que $\Sigma_{j=-\infty}^{\infty} \gamma_{k j}^{2}<\infty$. La normalité du processus n'est pas requise. Cependant, l'hypothèse sur les cumulants d'ordre 4 entraîne que la distribution du processus ne peut s'éloigner trop de la distribution normale. Posons pour $k=1,2$, et pour $j=0,1, \ldots, J$,

où

$$
c_{k,-j}=c_{k j}=\frac{1}{n_{k}} \sum_{t=1}^{n_{k}-j}\left(X_{k t}-\bar{X}_{k}\right)\left(X_{k, t+j}-\bar{X}_{k}\right),
$$

$$
\begin{gathered}
\bar{X}_{k}=\frac{1}{n_{k}} \sum_{t=1}^{n_{k}} X_{k t}, \quad \mathbf{c}_{k}=\left(c_{k 0}, c_{k 1}, \ldots, c_{k J}\right)^{\prime}, \\
\boldsymbol{\gamma}_{k}=\left(\gamma_{k 0}, \gamma_{k 1}, \ldots, \gamma_{k J}\right)^{\prime}, \quad \boldsymbol{\Sigma}_{k}=\lim _{n_{k} \rightarrow \infty} n_{k} \mathscr{E}\left[\left(\mathbf{c}_{k}-\boldsymbol{\gamma}_{k}\right)\left(\mathbf{c}_{k}-\boldsymbol{\gamma}_{k}\right)^{\prime}\right]
\end{gathered}
$$

et l'on suppose que $\boldsymbol{\Sigma}_{k}$ est définie positive.

En supposant que les deux réalisations sont indépendantes, $\mathbf{c}_{1}$ et $\mathbf{c}_{2}$ sont deux vecteurs aléatoires indépendants. La distribution asymptotique de $\mathbf{c}_{1}-\mathbf{c}_{2}$ est $\mathbf{N}\left(\boldsymbol{\gamma}_{1}-\boldsymbol{\gamma}_{2}\right.$, $n_{1}^{-1} \Sigma_{1}+n_{2}^{-1} \Sigma_{2}$ ). On désire tester l'hypothèse

$$
H_{0}: \gamma_{1 j}=\gamma_{2 j}, \quad j \geq 0 .
$$

Sous cette hypothèse, $\boldsymbol{\Sigma}_{1}=\boldsymbol{\Sigma}_{2}=\boldsymbol{\Sigma}$, d'où

$$
\mathbf{c}_{1}-\mathbf{c}_{2} \sim \mathrm{N}\left(0,\left(n_{1}+n_{2}\right)\left(n_{1} n_{2}\right)^{-1} \Sigma\right) \text {. }
$$


On pose $n=n_{1}$ et on suppose que $n \rightarrow \infty$ et que le rapport $n_{1} / n_{2}$ est constant. Soit

$$
\mathbf{z}^{(n)}=\left(\frac{n_{1} n_{2}}{n_{1}+n_{2}}\right)^{\frac{1}{2}}\left(\mathbf{c}_{1}-\mathbf{c}_{2}\right) \text {, }
$$

on a dès lors

$$
\mathbf{z}^{(n)} \stackrel{\mathscr{L}}{\rightarrow} \mathbf{z}, \quad \mathbf{z}^{(n)^{\prime}} \boldsymbol{\Sigma}^{-1} \mathbf{z}^{(n)} \stackrel{\mathscr{L}}{\rightarrow} \mathbf{z}^{\prime} \boldsymbol{\Sigma}^{-1} \mathbf{z}
$$

où $\mathbf{z}$ est $\mathbf{N}(0, \boldsymbol{\Sigma})$ et $\mathbf{z}^{\prime} \boldsymbol{\Sigma}^{-1} \mathbf{z}$ est $\chi_{j+1}^{2}$. La forme quadratique $\mathbf{z}^{(n)^{\prime}} \boldsymbol{\Sigma}^{-1} \mathbf{z}^{(n)}$ est une statistique similaire à celle utilisée pour effectuer des tests sur le vecteur moyenne d'une population multivariée.

On fournira dans la section suivante un estimateur convergent (en probabilité) de $\boldsymbol{\Sigma}_{k}$, soit $\hat{\boldsymbol{\Sigma}}_{k}$. Dès lors

$$
\hat{\mathbf{\Sigma}}^{(n)}=\left(n_{1}+n_{2}\right)^{-1}\left(n_{1} \hat{\mathbf{\Sigma}}_{1}+n_{2} \hat{\mathbf{\Sigma}}_{2}\right)
$$

est un estimateur convergent de $\boldsymbol{\Sigma}$ et $\left(\hat{\mathbf{\Sigma}}^{(n)}\right)^{-1}$ est aussi un estimateur convergent de $\boldsymbol{\Sigma}^{-1}$. Puisque

$$
\mathbf{z}^{(n)} \stackrel{\mathscr{L}}{\rightarrow} \mathbf{z}, \quad\left(\hat{\mathbf{\Sigma}}^{(n)}\right)^{-1} \stackrel{P}{\rightarrow} \mathbf{\Sigma}^{-1},
$$

par le Théorème 4.4 de Billingsley (1968), nous avons

$$
\left(\mathbf{z}^{(n)},\left(\hat{\mathbf{\Sigma}}^{(n)}\right)^{-1}\right) \stackrel{\mathscr{G}}{\rightarrow}\left(\mathbf{z}, \mathbf{\Sigma}^{-1}\right) .
$$

Définissons la fonction

$$
Q=Q\left(\mathbf{z}, \mathbf{\Sigma}^{-1}\right)=\mathbf{z}^{\prime} \mathbf{\Sigma}^{-1} \mathbf{z}
$$

qui est continue en chacun des éléments de $\mathbf{z}$ et de $\Sigma^{-1}$. Par le Théorème 5.1 de Billingsley, il découle que

$$
Q^{(n)}=Q\left(\mathbf{z}^{(n)},\left(\hat{\mathbf{\Sigma}}^{(n)}\right)^{-1}\right) \stackrel{\mathscr{L}}{\rightarrow} Q\left(\mathbf{z}, \mathbf{\Sigma}^{-1}\right)
$$

et comme nous avons montré déjà que $Q$ suit une loi $\chi_{J+1}^{2}$, il résulte que

$$
Q^{(n)} \stackrel{\mathscr{L}}{\rightarrow} \chi_{J+1}^{2}
$$

Nous proposons de tester $H_{0}$ en utilisant la statistique $Q^{(n)}$. L'hypothèse nulle est rejetée pour les grandes valeurs de $Q^{(n)}$ et les points critiques sont déterminés à l'aide de la distribution limite khi-deux.

\section{ESTIMATEUR DE LA COVARIANCE DE DEUX AUTOCOVARIANCES ÉCHANTILLONNALES}

Il s'agit d'obtenir un estimateur convergent $\hat{\boldsymbol{\Sigma}}$ de

$$
\Sigma=\lim _{n \rightarrow \infty} n \mathscr{E}\left[(\mathbf{c}-\boldsymbol{\gamma})(\mathbf{c}-\boldsymbol{\gamma})^{\prime}\right]
$$

où $\mathbf{c}=\left(c_{0}, c_{1}, \ldots, c_{J}\right)^{\prime}$ est calculé par une formule similaire à (2.1) à partir d'une réalisation finie de longeur $n$ d'un processus stationnaire $X$ de moments d'ordre 1 et 2

$$
\mu=\mathscr{E}\left[X_{t}\right], \quad \gamma_{j}=\mathscr{E}\left[\left(X_{t}-\mu\right)\left(X_{t+j}-\mu\right)\right]
$$

dont tous les cumulants d'ordre 4 sont nuls et tel que $\Sigma_{j=-\infty}^{\infty} \gamma_{j}^{2}<\infty$. On sait que l'élément $(l, i) l, i=0,1, \ldots, J$, de $\Sigma$ est de la forme (Anderson 1971, p. 481)

$$
\sigma_{l i}=\theta_{i-l}+\theta_{i+l}
$$


où

$$
\theta_{i}=\sum_{j=-\infty}^{\infty} \gamma_{j} \gamma_{i+j}
$$

La matrice $\Sigma$ est définie non négative, quelle que soit la fonction d'autocovariance $\gamma_{j}, j$ entier. Robinson (1977) a proposé l'estimateur suivante de $\boldsymbol{\theta}_{i}$ :

$$
h_{i}=\sum_{j}^{\prime} w\left(j b_{n}\right) c_{j} c_{i+j}
$$

où la somme " ' " est effectuée pour $-n+1 \leq j \leq n-i-1$. Si $|w(0)|=1,|w(\cdot)|$ est une fonction intégrable, continue à l'origine et possédant au plus un nombre fini de discontinuités et si la suite $b_{n}$ décroit vers 0 de telle façon que $n b_{n} \rightarrow \infty$ lorsque $n \rightarrow \infty$, alors $\mathscr{E}\left[\left|h_{i}-\theta_{i}\right|\right] \rightarrow 0$, ce qui entraîne la convergence en probabilité de $h_{i}$ vers $\theta_{i}$. Cependant, il n'est pas assuré que la matrice $\hat{\mathbf{\Sigma}}=\left(\hat{\boldsymbol{\sigma}}_{l i}\right), l, i=0,1, \ldots, J$ avec $\hat{\boldsymbol{\sigma}}_{l i}=h_{i-l}+h_{i+l}$ soit définie positive.

Au lieu d'utiliser l'estimateur $h_{i}$, nous proposons

$$
g_{i}=\sum_{j}^{\prime} w\left(j b_{n}\right) w\left((i+j) b_{n}\right) c_{j} c_{i+j}
$$

avec les mêmes hypothèses que Robinson sauf que $w^{2}(\cdot)$ est supposée être une fonction intégrable et la fonction de $j$ entier, $w_{j}=w\left(j b_{n}\right)$ est supposée être une fonction définie positive. De telles fonctions existent, par exemple la fenêtre de Bartlett modifiée (Anderson 1971, p. 512) utilisée dans l'estimation de la densité spectrale. On sait alors (voir Loève 1978, p. 134) que la fonction de $j$ entier, $w_{j} c_{j}$ est aussi une fonction d'autocovariance de sorte qu'en substituant $w_{j} c_{j}$ à $\gamma_{j}$ dans (3.2), on obtient une matrice $\hat{\mathbf{\Sigma}}$ définie positive. Il reste à montrer la convergence de $g_{i}$ vers $\theta_{i}$ au sens de la norme $L_{1}$. Ceci peut se faire en adaptant la démonstration de Robinson. Dans la première partie de celle-ci, Robinson suppose $\mu=0$ et envisage

$$
\tilde{c}_{j}=\frac{1}{n} \sum_{t=1}^{n-j} X_{t} X_{t+j}
$$

comme estimateur de $\gamma_{j}$. Il montre ensuite qu'en définissant $c_{j}$ par

$$
\frac{1}{n} \sum_{t=1}^{n-j} X_{t} X_{t+j}-\bar{X}^{2}
$$

on n'introduit pas de différence asymptotiquement. Cette partie du raisonnement doit également être modifiée car la fonction $c_{j}, j$ entier, décrite par (3.6) n'est pa définie positive. Une démonstration plus courte, en une seule étape et tenant compte des différences mentionnées ci-dessus suit. Dans la démonstration du théorème, nous utiliserons le lemme suivant.

LEMME 1. Soit $X_{1}, \ldots, X_{n}$ une réalisation finie d' un processus stationnaire dont les deux premiers moments sont notés selon (3.1) dont tous les cumulants d'ordre 4 sont nuls et tel que $\Sigma_{j=-\infty}^{\infty} \gamma_{j}^{2}<\infty$. Soit $c_{j}$ l'autocovariance échantillonnale de délai $j$ définie par une formule similaire $\grave{a}(2.1)$ et posons $\tilde{\gamma}_{j}=(1-|j| / n) \gamma_{j},|j| \leq n-1$. Alors $\mathscr{E}\left[\left(c_{j}-\tilde{\gamma}_{j}\right)^{2}\right]=O(1 / n)$ uniformément en $j$.

Démonstration. Sans perte de généralité, nous pouvons poser $\mu=0$ et soit $\tilde{c}_{j}$ telle que définie par (3.5). Montrons dans un premier temps que $\mathscr{E}\left[\left(c_{j}-\tilde{c}_{j}\right)^{2}\right]=O(1 / n)$ uniformément en $j$. D'abord nous avons 


$$
c_{j}-\tilde{c}_{j}=-\left(1+\frac{j}{n}\right) \bar{X}^{2}+\frac{\bar{X}}{n} \sum_{j}^{*} X_{t}
$$

où la somme "*" est effectuée pour $1 \leq t \leq j$ et pour $n-j<t \leq n$, d'où il s'en suit que

$$
\left(c_{j}-\tilde{c}_{j}\right)^{2} \leq 2\left(4 \bar{X}^{4}+B\right)
$$

avec

$$
B=\frac{\bar{X}^{2}}{n^{2}}\left(\sum_{t}^{*} X_{t}\right)^{2}=\frac{1}{n^{4}} \sum_{h} \sum_{k} \sum_{t}^{*} \sum_{s}^{*} X_{h} X_{k} X_{t} X_{s}
$$

(lorsque les limites de sommation de $\Sigma$ sont omises, la somme porte sur les valeurs $1, \ldots, n)$. En utilisant la formule d'Isserlis (Priestley 1981, vol. 1, p. 325), on vérifie que $\mathscr{E}[B]=O(1 / n)$ uniformément en $j$. De plus, par Robinson $(1977$, p. 239$), \mathscr{E}\left[\bar{X}^{4}\right]=$ $O(1 / n)$ et il s'en suit que $\mathscr{E}\left[\left(c_{j}-\tilde{c}_{j}\right)^{2}\right]=O(1 / n)$ uniformément en $j$. Finalement, le résultat énoncé est obtenu de l'inégalité

$$
\mathscr{E}\left[\left(c_{j}-\tilde{\gamma}_{j}\right)^{2}\right] \leq 2\left\{\mathscr{E}\left[\left(c_{j}-\tilde{c}_{j}\right)^{2}\right]+\mathscr{E}\left[\left(\tilde{c}_{j}-\tilde{\gamma}_{j}\right)^{2}\right]\right\}
$$

puisque $\mathscr{E}\left[\left(c_{j}-\tilde{\gamma}_{j}\right)^{2}\right]=O(1 / n)$ uniformément en $j$ d'après Robinson $(1977$, p. 238).

THÉOREME 1. Soit $w: \mathbb{R} \rightarrow \mathbb{R}$ une fonction continue à l'origine, bornée, possédant au plus un nombre fini de discontinuités et telle que $w^{2}(0)=1, w^{2}(\cdot)$ est intégrable. De plus soit $b_{n}, n \geq 1$, une suite de nombres réels tels que $b_{n} \downarrow 0, n b_{n} \rightarrow \infty$ lorsque $n \rightarrow \infty$. Alors sous les hypothèses du Lemme 1, l'estimateur $g_{i}$ défini par (3.4) converge vers $\theta_{i}$ au sens de la norme $L_{1}$.

Démonstration. Nous pouvons écrire

$$
g_{i}-\theta_{i}=d_{1}+d_{2}+d_{3}+d_{4}
$$

où $\tilde{\gamma}_{j}$ est définie de la même façon que précédement et

$$
\begin{aligned}
& d_{1}=\sum_{j}^{\prime} w\left(j b_{n}\right) w\left((i+j) b_{n}\right)\left(c_{j}-\tilde{\gamma}_{j}\right) c_{i+j}, \\
& d_{2}=\sum_{j}^{\prime} w\left(j b_{n}\right) w\left((i+j) b_{n}\right) \tilde{\gamma}_{j}\left(c_{i+j}-\tilde{\gamma}_{i+j}\right), \\
& d_{3}=\sum_{j}^{\prime}\left\{w\left(j b_{n}\right) w\left((i+j) b_{n}\right)-1\right\} \tilde{\gamma}_{j} \tilde{\gamma}_{i+j}, \\
& d_{4}=\sum_{j}^{\prime} \tilde{\gamma}_{j} \tilde{\gamma}_{i+j}-\theta_{i} .
\end{aligned}
$$

Par l'inégalité de Cauchy-Schwarz, nous obtenons

$$
\mathscr{E}\left[\left|g_{i}-\theta_{i}\right|\right] \leq\left(\mathscr{E}\left[e_{1}\right] \mathscr{E}\left[e_{2}\right]\right)^{\frac{1}{2}}+\left(e_{3} \mathscr{E}\left[e_{4}\right]\right)^{\frac{1}{2}}+\left(e_{5} e_{6}\right)^{\frac{1}{2}}+\left|d_{4}\right|
$$

où

$$
\begin{array}{ll}
e_{1}=\sum_{j}^{\prime} w^{2}\left(j b_{n}\right)\left(c_{j}-\tilde{\gamma}_{j}\right)^{2}, & e_{2}=\sum_{j}^{\prime} w^{2}\left((i+j) b_{n}\right) c_{i+j}^{2}, \\
e_{3}=\sum_{j}^{\prime} w^{2}\left(j b_{n}\right) \tilde{\gamma}_{j}^{2}, & e_{4}=\sum_{j}^{\prime} w^{2}\left((i+j) b_{n}\right)\left(c_{i+j}-\tilde{\gamma}_{i+j}\right)^{2},
\end{array}
$$




$$
e_{5}=\sum_{j}^{\prime}\left|w\left(j b_{n}\right) w\left((i+j) b_{n}\right)-1\right| \tilde{\gamma}_{j}^{2}, \quad e_{6}=\sum_{j}^{\prime}\left|w\left(j b_{n}\right) w\left((i+j) b_{n}\right)-1\right| \tilde{\gamma}_{i+j}^{2} .
$$

Nous allons montrer que chacun des termes de (3.7) tend vers 0 lorsque $n \rightarrow \infty$.

D'abord le Lemme 1 entraîne que

$$
\mathscr{E}\left[e_{1}\right] \leq O\left(n^{-1}\right) \sum_{j}^{\prime} w^{2}\left(j b_{n}\right)=O\left(n^{-1} b_{n}^{-1}\right) b_{n} \sum_{j}^{\prime} w^{2}\left(j b_{n}\right)
$$

et avec les hypothèses faites sur la fonction $w(\cdot)$, il s'en suit que

$$
\lim _{n \rightarrow \infty} b_{n} \sum_{j}^{\prime} w^{2}\left(j b_{n}\right)=\int_{-\infty}^{\infty} w^{2}(z) d z<\infty
$$

et donc $\mathscr{E}\left[e_{1}\right] \rightarrow 0$ puisque $n b_{n} \rightarrow \infty$. De même, $\mathscr{E}\left[e_{4}\right] \rightarrow 0$.

Montrons maintenant que $e_{2}$ et $e_{3}$ sont bornés. Puisque $w(\cdot)$ est une fonction bornée, $e_{3} \leq\left\{\sup _{z \in \mathbb{R}} w^{2}(z)\right\} \Sigma_{j}^{\prime} \tilde{\gamma}_{j}^{2}<\infty \operatorname{car} \Sigma_{j}^{\prime} \tilde{\gamma}_{j}^{2}<\Sigma_{j}^{\prime} \gamma_{j}^{2} \leq \theta_{0}<\infty$. De plus, nous pouvons écrire

$$
\mathscr{E}\left[e_{2}\right] \leq 2 \sum_{j}^{\prime} w^{2}\left((i+j) b_{n}\right)\left\{\mathscr{E}\left[\left(c_{i+j}-\tilde{\gamma}_{i+j}\right)^{2}\right]+\gamma_{i+j}^{2}\right\}=2\left(\mathscr{E}\left[e_{4}\right]+e_{3}^{\prime}\right)<\infty
$$

où $e_{3}^{\prime}$ est un terme borné similaire à $e_{3}$.

Décomposons le terme $e_{5}$ de la façon suivante: pour $l$ fixé, $e_{5}=s_{1}+s_{2}$ où $s_{1}$ représente la somme pour $|j| \leq l$ et $s_{2}$ la somme pour $|j|>l$. D'abord pour $|j| \leq l, j b_{n} \rightarrow 0$, $(i+j) b_{n} \rightarrow 0$ et $w(\cdot)$ étant continue à l'origine, on a $w\left(j b_{n}\right) w\left((i+j) b_{n}\right) \rightarrow w^{2}(0)=1$ et donc $s_{1} \rightarrow 0$. De plus, on peut écrire

$$
s_{2} \leq\left\{\left(\sup _{z \in k}|w(z)|\right)^{2}+1\right\} \sum_{|j|>1} \gamma_{j}^{2}
$$

et $\Sigma_{|j|>1} \gamma_{j}^{2}$ peut être fait arbitrairement petit puisque $\theta_{0}<\infty$. D'où $s_{2} \rightarrow 0$ et $e_{5} \rightarrow 0$. Par similitude, $e_{6} \rightarrow 0$.

Finalement, on peut écrire

$$
\left|d_{4}\right| \leq\left|\sum_{j}^{\prime}\left(\tilde{\gamma}_{j} \tilde{\gamma}_{i+j}-\gamma_{i} \gamma_{i+j}\right)\right|+\left|\sum_{j=-\infty}^{-n} \gamma_{j} \gamma_{i+j}\right|+\left|\sum_{j=n-i}^{\tilde{n}} \gamma_{j} \gamma_{i+j}\right| .
$$

Les deux derniers termes tendant vers 0 puisque la série définissant $\theta_{i}$ converge. Le premier terme tend aussi vers 0 (Robinson 1977, p. 239). En rassemblant les résultats, la démonstration est complétée.

\section{RESULTATS DE SIMULATION}

Les sections précédentes contiennent trois types de résultats asymptotiques: la convergence en probabilité d'un estimateur de $\boldsymbol{\Sigma}$, la distribution limite de $\mathbf{c}_{1}-\mathbf{c}_{2}$ et la distribution limite de $Q^{(n)}$. Pour des raisons de commodité, l'étude sur des séries finies qui fait l'objet de cette section est limitée au dernier cas. Des simulation de Monte Carlo sont employées pour examiner le comportement du test de l'hypothèse $H_{0}: \gamma_{1 j}=\gamma_{2 j}, j \geq 0$, qui est basé sur la distribution limite de $Q^{(n)}$. Des couples de séries de longeurs 50, 100 et 200 ont été engendrées à partir de processus autorégressifs d'ordre 1 (AR) où de processus de moyenne mobile d'ordre 1 (MA) décrits dans le Tableau 1. Les innovations $a_{t}$ sont distribuées selon une loi normale centrée réduite. La simulation a été réalisée à l'aide du générateur de nombres pseudoaléatoires GGUBS de la librairie IMSL (1980). 
TABLEAU 1: Les quatre processus utilisés lors de l'étude de Monte-Carlo.

\begin{tabular}{lcrrrrr}
\hline \hline & & \multicolumn{5}{c}{ Fonction d'autocovariance } \\
\cline { 3 - 7 } Nom & Equation & $\gamma_{0}$ & \multicolumn{1}{c}{$\gamma_{1}$} & $\gamma_{2}$ & $\gamma_{3}$ & $\gamma_{4}$ \\
\hline $\operatorname{AR}(0.2)$ & $X_{1}=0.2 X_{t-1}+a_{t}$ & 1.04 & 0.21 & 0.04 & 0.01 & 0.00 \\
$\operatorname{AR}(0.8)$ & $X_{1}=0.8 X_{t-1}+a_{t}$ & 2.78 & 2.22 & 1.78 & 1.42 & 1.14 \\
$\operatorname{MA}(0.2)$ & $X_{t}=a_{1}-0.2 a_{t-1}$ & 1.04 & -0.20 & 0.00 & 0.00 & 0.00 \\
$\operatorname{MA}(0.8)$ & $X_{t}=a_{t}-0.8 a_{t-1}$ & 1.64 & -0.80 & 0.00 & 0.00 & 0.00 \\
\hline
\end{tabular}

TABLEAU 2: Valeurs de $b_{n, H}$.

\begin{tabular}{cccc}
\hline \hline$H$ & $n=50$ & 100 & 200 \\
\hline 1 & 7 & 10 & 14 \\
3 & 21 & 30 & 42 \\
5 & 35 & 50 & 70 \\
\hline
\end{tabular}

TABLEAU 3: Valeur moyenne des probabilités de signification $\alpha$ sous $H_{0}$ (à comparer à 0.5 ). Le symbole + indique que la moyenne des $\alpha$ ne s'écarte pas significativement de la valeur attendue 0.5 au niveau de probabilité 0.01 .

\begin{tabular}{|c|c|c|c|c|c|c|c|}
\hline \multirow[b]{2}{*}{$H$} & \multirow[b]{2}{*}{$n$} & \multicolumn{3}{|c|}{$\operatorname{AR}(0.2)$} & \multicolumn{3}{|c|}{$\operatorname{AR}(0.8)$} \\
\hline & & $J=1$ & 2 & 4 & 1 & 2 & 4 \\
\hline \multirow[t]{3}{*}{1} & 50 & $0.50^{+}$ & $0.53^{+}$ & 0.56 & $0.53^{+}$ & 0.56 & 0.58 \\
\hline & 100 & $0.52^{+}$ & 0.54 & 0.56 & $0.51^{+}$ & 0.54 & 0.56 \\
\hline & 200 & $0.50^{+}$ & $0.52^{+}$ & 0.53 & $0.52^{+}$ & 0.54 & 0.56 \\
\hline \multirow[t]{3}{*}{3} & 50 & $0.52^{+}$ & 0.56 & 0.59 & 0.58 & 0.60 & 0.62 \\
\hline & 100 & $0.53^{+}$ & 0.56 & 0.58 & 0.57 & 0.58 & 0.60 \\
\hline & 200 & $0.49^{+}$ & $0.51^{+}$ & 0.53 & 0.56 & 0.57 & 0.58 \\
\hline \multirow[t]{4}{*}{5} & 50 & 0.58 & 0.63 & 0.67 & 0.69 & 0.69 & 0.70 \\
\hline & 100 & 0.57 & 0.61 & 0.64 & 0.68 & 0.67 & 0.68 \\
\hline & 200 & $0.53^{+}$ & 0.56 & 0.58 & 0.62 & 0.60 & 0.61 \\
\hline & & \multicolumn{3}{|c|}{$\operatorname{MA}(0.2)$} & \multicolumn{3}{|c|}{ MA(0.8) } \\
\hline$H$ & $n$ & $J=1$ & 2 & 4 & 1 & 2 & 4 \\
\hline \multirow[t]{3}{*}{1} & 50 & $0.53^{+}$ & 0.56 & 0.59 & 0.55 & 0.58 & 0.60 \\
\hline & 100 & $0.50^{+}$ & $0.53^{+}$ & 0.55 & 0.54 & 0.56 & 0.58 \\
\hline & 200 & $0.50^{+}$ & $0.52^{+}$ & 0.54 & 0.55 & 0.56 & 0.58 \\
\hline \multirow[t]{3}{*}{3} & 50 & 0.55 & 0.59 & 0.62 & 0.60 & 0.61 & 0.64 \\
\hline & 100 & $0.51^{+}$ & 0.54 & 0.57 & 0.56 & 0.58 & 0.60 \\
\hline & 200 & $0.50^{+}$ & $0.52^{+}$ & 0.54 & 0.56 & 0.57 & 0.59 \\
\hline \multirow[t]{3}{*}{5} & 50 & 0.61 & 0.66 & 0.69 & 0.68 & 0.69 & 0.71 \\
\hline & 100 & $0.53^{+}$ & 0.57 & 0.61 & 0.62 & 0.63 & 0.65 \\
\hline & 200 & $0.52^{+}$ & 0.55 & 0.58 & 0.60 & 0.60 & 0.61 \\
\hline
\end{tabular}


TABLEAU 4: Risque de premier type (\%) pour trois niveaux nominaux $\alpha(\%)$ du test avec $H=1$.

\begin{tabular}{|c|c|c|c|c|c|c|c|}
\hline \multirow[b]{2}{*}{$\alpha$} & \multirow[b]{2}{*}{$n$} & \multicolumn{3}{|c|}{$\operatorname{AR}(0.2)$} & \multicolumn{3}{|c|}{$\operatorname{AR}(0.8)$} \\
\hline & & $J=1$ & 2 & 4 & 1 & 2 & 4 \\
\hline \multirow[t]{3}{*}{5} & 50 & 1.6 & 0.4 & 0.4 & 0.8 & 0.0 & 0.0 \\
\hline & 100 & 4.4 & 3.6 & 1.6 & 1.6 & 0.0 & 0.0 \\
\hline & 200 & 4.0 & 3.2 & 2.4 & 2.8 & 1.2 & 0.8 \\
\hline \multirow[t]{3}{*}{10} & 50 & 5.2 & 3.6 & 1.2 & 3.6 & 1.6 & 1.2 \\
\hline & 100 & 8.4 & 6.0 & 4.4 & 6.4 & 4.4 & 3.2 \\
\hline & 200 & 11.2 & 9.6 & 7.2 & 7.2 & 5.2 & 3.6 \\
\hline \multirow[t]{4}{*}{25} & 50 & 24.8 & 20.4 & 17.2 & 22.0 & 13.6 & 10.8 \\
\hline & 100 & 22.8 & 20.0 & 18.4 & 22.8 & 20.0 & 17.6 \\
\hline & 200 & 27.6 & 24.0 & 20.0 & 18.8 & 17.2 & 15.6 \\
\hline & & \multicolumn{3}{|c|}{$\operatorname{MA}(0.2)$} & \multicolumn{3}{|c|}{$\operatorname{MA}(0.8)$} \\
\hline$\alpha$ & $n$ & $J=1$ & 2 & 4 & 1 & 2 & 4 \\
\hline \multirow[t]{3}{*}{5} & 50 & 0.8 & 0.4 & 0.4 & 0.8 & 0.4 & 0.4 \\
\hline & 100 & 2.4 & 0.8 & 0.8 & 2.4 & 2.0 & 1.2 \\
\hline & 200 & 1.6 & 1.2 & 1.2 & 2.8 & 1.6 & 1.2 \\
\hline \multirow[t]{3}{*}{10} & 50 & 2.4 & 1.2 & 0.8 & 2.8 & 2.0 & 1.2 \\
\hline & 100 & 6.4 & 4.0 & 3.6 & 6.0 & 4.4 & 2.8 \\
\hline & 200 & 8.4 & 5.6 & 4.8 & 5.6 & 4.4 & 4.0 \\
\hline \multirow[t]{3}{*}{25} & 50 & 19.2 & 14.8 & 10.4 & 15.6 & 11.6 & 8.0 \\
\hline & 100 & 25.2 & 21.6 & 17.2 & 20.8 & 16.8 & 13.2 \\
\hline & 200 & 23.6 & 20.4 & 18.8 & 19.2 & 16.0 & 14.8 \\
\hline
\end{tabular}

Le test de $H_{0}$ a été effectué pour chaque couple de processus du Tableau 1. Les quatres couples de processus identiques servent à examiner le biais du test. Les autres couples permettent d'apprécier la puissance du test. Pour chacun des 10 couples de processus, 500 réalisations indépendantes, de longeur 200, ont été générées. Les 50 premières, les 100 premières et les 200 observations ont été employées successivement afin de déterminer l'influence sur les résultats de la longeur des deux séries $n=n_{1}=n_{2}$. L'ordre maximum $J$ des autocovariances utilisées dans les vecteurs $c_{1}$ et $c_{2}$ a été pris égal à 1,2 et 4 . La fonction $w(\cdot)$ utilisée dans l'estimation des $\sigma_{l i}$ est la fenêtre de Bartlett modifiée. Les poids $w\left(j b_{n}\right)$ de la formule (3.4) que nous notons par $w_{j}\left(b_{n, H}\right)$ sont définis comme suit:

$$
w_{j}\left(b_{n, H}\right)= \begin{cases}1-\frac{|j|}{b_{n, H}}, & |j| \leq b_{n, H}, \\ 0, & |j|>b_{n, H},\end{cases}
$$

où $b_{n, H}$ a été choisi, en conformité avec les conditions exprimées dans le Théorème 1 comme étant de la forme $b_{n, H}=H n^{\frac{1}{2}}$ avec trois valeurs différentes pour $H: H=1,3$ ou 5 . Le Tableau 2 donne les valeurs utilisées pour $b_{n, H}$.

Les résultats des simulations sont présentés dans les deux interprétations possibles des tests. Pour chaque couple de séries simulées, on détermine la probabilité de signification du test $\alpha=P\left[\chi_{j+1}^{2}>Q^{(n)}\right]$. On examine alors la distribution de $\alpha$ à travers les 500 réalisations. Dans la première interprétation, si l'hypothèse nulle $H_{0}$ est vraie, et supposant la loi limite valable pour $n$ fini, on sait que $\alpha$ doit être distribué selon une loi uniforme sur $[0,1]$, donc de moyenne 0.5 et d'écart-type 0.289 . Par conséquent, on a étudié la 
TABLEaU 5: Puissance du test (\%) aux niveaux de signification $5 \%$ et $10 \%$ pour les six couples de processus. $H=1$ et la distance $\delta$ entre les processus est donnée entre parenthèses.

\begin{tabular}{|c|c|c|c|c|c|c|c|}
\hline \multirow[b]{2}{*}{ Couple de processus } & \multirow[b]{2}{*}{$n$} & \multicolumn{3}{|c|}{$5 \%$} & \multicolumn{3}{|c|}{$10 \%$} \\
\hline & & $J=1$ & 2 & 4 & 1 & 2 & 4 \\
\hline \multirow[t]{4}{*}{$\operatorname{AR}(0.2)-\operatorname{AR}(0.8)$} & & (2.7) & (3.2) & (3.7) & $(2.7)$ & (3.2) & (3.7) \\
\hline & 50 & 66 & 32 & 22 & 86 & 60 & 45 \\
\hline & 100 & 98 & 96 & 93 & 100 & 99 & 98 \\
\hline & 200 & 100 & 100 & 100 & 100 & 100 & 100 \\
\hline \multirow[t]{4}{*}{$\operatorname{AR}(0.2)-\mathrm{MA}(0.2)$} & & $(0.4)$ & $(0.4)$ & $(0.4)$ & $(0.4)$ & $(0.4)$ & $(0.4)$ \\
\hline & 50 & 21 & 11 & 7 & 42 & 29 & 20 \\
\hline & 100 & 59 & 50 & 43 & 74 & 68 & 62 \\
\hline & 200 & 97 & 96 & 96 & 99 & 98 & 98 \\
\hline \multirow[t]{4}{*}{$\operatorname{AR}(0.2)-\mathrm{MA}(0.8)$} & & $(1.2)$ & (1.2) & (1.2) & $(1.2)$ & (1.2) & (1.2) \\
\hline & 50 & 87 & 74 & 64 & 96 & 92 & 88 \\
\hline & 100 & 100 & 100 & 100 & 100 & 100 & 100 \\
\hline & 200 & 100 & 100 & 100 & 100 & 100 & 100 \\
\hline \multirow[t]{4}{*}{$\operatorname{AR}(0.8)-\mathrm{MA}(0.2)$} & & (3.0) & (3.5) & (3.9) & (3.0) & (3.5) & (3.9) \\
\hline & 50 & 98 & 85 & 73 & 99 & 98 & 95 \\
\hline & 100 & 100 & 100 & 100 & 100 & 100 & 100 \\
\hline & 200 & 100 & 100 & 100 & 100 & 100 & 100 \\
\hline \multirow[t]{4}{*}{$\operatorname{AR}(0.8)-\mathrm{MA}(0.8)$} & & (3.2) & (3.7) & (4.1) & (3.2) & (3.7) & (4.1) \\
\hline & 50 & 100 & 99 & 98 & 100 & 100 & 100 \\
\hline & 100 & 100 & 100 & 100 & 100 & 100 & 100 \\
\hline & 200 & 100 & 100 & 100 & 100 & 100 & 100 \\
\hline \multirow[t]{4}{*}{$\operatorname{MA}(0.2)-\operatorname{MA}(0.8)$} & & $(0.8)$ & $(0.8)$ & $(0.8)$ & $(0.8)$ & $(0.8)$ & $(0.8)$ \\
\hline & 50 & 17 & 8 & 5 & 38 & 25 & 17 \\
\hline & 100 & 57 & 50 & 42 & 72 & 66 & 60 \\
\hline & 200 & 92 & 91 & 90 & 97 & 96 & 95 \\
\hline
\end{tabular}

moyenne et l'écart-type de la distribution observée des valeurs de $\alpha$ qui doivent être comparés aux moments théoriques donnés ci-dessus pour les quatre couples de processus identiques. Un écart de la moyenne dans un sens ou dans l'autre indique que le test est biaisé. Dans la deuxième interprétation des tests, on détermine parmi les 500 réalisations le pourcentage de rejets de $H_{0}$ aux niveaux de probabilité nominaux de $5 \%, 10 \%$ et $25 \%$. Pour les quatre couples de processus identiques, ces pourcentages devraient avoisiner les niveaux nominaux. Pour les six couples de processus différents, ils doivent être aussi proches de $100 \%$ que possible. L'analyse des résultats est effectuée en deux temps: on examine d'abord le biais du test en fonction de $n$ et de $J$, ensuite la puissance du test.

Le Tableau 3 montre les valeurs moyennes de $\alpha$ pour les quatre couples de processus identiques. Les meilleurs résultats sont obtenus pour $H=1$ et non pour $H=3$ ou $H=5$, aussi limiterons-nous la suite de la discussion à la fenêtre spécifiée par $H=1$. On observe que les tests avec $J=2$ ou 4 sont plus biaisés que ceux avec $J=1$. Le biais paraît moindre pour les processus autorégressifs que pour les processus de moyenne mobile. Il semble que la proximité de la frontière de stationnarité ou d'inversibilité, selon le cas, ait tendance à accroître le biais. La comparaison de l'écart-type des valeurs de $\alpha$ par rapport à la valeur attendue 0.289 suit un comportement similaire à celle de la moyenne, mais n'a pas été reprise ici.

Le Tableau 4 donne, pour $H=1$, le risque de premier type aux niveaux nominaux de $5 \%, 10 \%$ et $25 \%$. Les résultats confirment les constatations précédentes. Les résultats des tableaux 3 et 4 indiquent que le biais est une fonction décroissante de $n$. 
Le Tableau 5 fournit une évaluation de la puissance du test, effectué au niveau nominal de $5 \%$ ou $10 \%$. Intuitivement, on s'attend à ce que la puissance augmente avec $n$, avec $\alpha$ et avec la distance entre les deux processus, mesurée de façon appropriée. Nous avons utilisé ici la distance euclidienne $\delta=\left\{\sum_{j=0}^{J}\left(\gamma_{1 j}-\gamma_{2 j}\right)^{2}\right\}^{\frac{1}{2}}$. Le classement est bon dans l'ensemble, à l'exception des comparaisons $\operatorname{AR}(0.2)-\operatorname{MA}(0.2)$ avec $\operatorname{MA}(0.2)-\operatorname{MA}(0.8)$ et $\operatorname{AR}(0.2)-M A(0.8)$ avec $\operatorname{AR}(0.2)-\operatorname{AR}(0.8)$. Il doit s'agir d'une manifestation du biais, plus important avec les processus de moyenne mobile qu'avec les processus autorégressifs. La perte de puissance due à l'emploi d'une valeur de $J$ supérieure à 1 s'explique possiblement par le fait que les processus considérés sont caractérisés par leur coefficient d'autocorrélation de délai 1. Cependant, elle peut aussi être attribuée au biais qui est tel que pour un test de niveau $\alpha$, on doit souvent utiliser un niveau nominal plus grand.

\section{REMERCIEMENTS}

Les auteurs sont très reconnaissants à $M$. Normand Ranger de l'aide apportée lors des simulations.

\section{REFERENCES}

Anderson, T.W. (1971). The Statistical Analysis of Time Series. Wiley, New York.

Billingsley, P. (1968). Convergence of Probability Measures. Wiley, New York.

Box, G.E.P., and Jenkins, G.M. (1976). Time Series Analysis: Forecasting and Control. Edition revisée. Holden-Day, San Francisco.

Brillinger, D.R. (1978). Confidence intervals for the cross-covariance function. Selecta Statist. Canad., 5, $1-16$.

Brillinger, D.R. (1981). Time Series: Data Analysis and Theory. Edition revisée. Holden-Day, San Francisco. Loève, M. (1978). Probability Theory II. Fourth Edition, Springer-Verlag, New York.

Priesley, M.B. (1981). Spectral Analysis and Time Series. Volume 1. Academic Press, New York.

Robinson, P.M. (1977). Estimating variances and covariances of sample autocorrelations and autocovariances. Austral. J. Statist., 19, 236-240.

Taniguchi, M. (1982). On estimation of the integrals of the fourth order cumulant spectral density. Biometrika, $69,117-122$.

Received 30 September 1982

Revised 1 May 1984

Accepted 12 June 1984
Institute de statistique Université libre de Bruxelles

Campus Plaine - C.P. 210

Boulevard de Triomphe 1050 Bruxelles Belgique

Département d'informatique et de recherche opérationnelle Université de Montréal Casse Postale 6128, Succarsale "A" Montréal, P.Q. H3C 3J7

Canada 